\title{
Editorial
}

\section{Thankfulness to authors, reviewers, and readers}

\section{Microbes and Infectious Diseases Editorial Board Members}

With the second year of successful launching Microbes and Infectious Diseases, we've received 215 submissions from different countries (Africa: Egypt, Sudan, Tunisia, Senegal, Nigeria, Cameroon, Gabon, Tanzania, Burkina Faso and Benin; Asia: Saudi Arabia, Iraq, Iran, India, Pakistan, Bangladesh, Malaysia, Hong Kong, and China; Europe: Bosnia and Herzegovina, Turkey, Austria, and Italy; Latin America: Cuba and Brazil; and Australia). Although many of our papers are still in the peer review process, we have already published 99 papers online with a $45 \%$ rejection rate. The median time from submission to online publication is 38 days.

The Editorial Board Members have worked hard to meet the goals of Microbes and Infectious Diseases in delivering timely decisions and maintaining publication of high-quality papers through vigorous and scientific peer review process. Besides, we believe that authors' response to the valuable comments of our editors and reviewers is a cornerstone for impacting in the international research community.

We would like to introduce our deep thanks to 68 individual reviewers who give so freely of their time and expertise to critically evaluating manuscripts for the journal. Our reviewers, like our authors, come from all over the world to maintain a wide authorship, readership and global citation activity. It is noteworthy that within the first two years of our journal's age, authors and reviewers are representing over 30 countries.

The policies and guidelines of Microbes and Infectious Diseases would help secure sufficient quality and novelty of content to become competitive with our national, regional and international competitors.

We would also like to take this opportunity to acknowledge our reviewers more publicly, highlighting our most outstanding reviewers. Those reviewers who not only carry out many assignments but also demonstrate professionalism and provide comments that truly help the authors improve their work.

\section{Reviewers List (in alphabetical order):}

$\begin{array}{lr}\text { Abdulrahman Alfulaty } & \text { Nigeria } \\ \text { Abeer Aboelazm } & \text { Egypt } \\ \text { Abeer Ezat } & \text { Egypt } \\ \text { Abeer Nafee } & \text { Egypt } \\ \text { Ahmed Asaad } & \text { Egypt } \\ \text { Ahmed Cihad } & \text { Turkey } \\ \text { Ahmed Fakhr } & \text { Egypt } \\ \text { Ajay Prajapati } & \text { India }\end{array}$


Ali Alnasser

Amal algendy

Amal Atta

Amani El Kholy

Ayman Allam

Edmond PUCA

Eman Elbehedy

Essodolom Taale

Faiza Amber Siddiqui

Fatma Abd El-Aziz

Fatma Moharram

Gamil Zedan

Ghada Mahmoud M. Fathy

Hanaa Abd El-hady

Hani Mowafi

Hassan Alnasser

Hosam Abd Elaziz

Ibrahim Ogunyinka

James Oyeniyi

Jihan Shawky

Jihan El-Hadidy

Khaled Aboshanab

Laila Alkady

MabrouK Ghonaim

Mahmoud Tawfick

Mai Maamoun

Majed Wakid

Makwin makut

Manal Bahgat

Matthew Okon

Mahmoud Abd Elsabour
Saudi Arabia

Egypt

Egypt

Egypt

Egypt

Albania

Egypt

Togo

USA

Egypt

Egypt

Egypt

Egypt

Egypt

Egypt

Saudi Arabia

Egypt

Nigeria

Nigeria

Egypt

Egypt

Egypt

Egypt

Egypt

Egypt

Egypt

Saudi Arabia

Nigeria

Egypt

Nigeria

Egypt
Mahmoud Shoukry

Maysa Abdallah Saeed

Mervat Hesham

Moawia Elbadri

Mohammad Abdullah

Mohammad Azizur

Mohammad Basharat

Mohamed EIFeky

Mohamed Al sweify

Mohamed Ansar

Mohamed Mortada

Mohamed Sarhan

Mohsen Hassan

Mona Aboserea

Muhammad Salihu

Nahed Mostafa

Neveen antouny

Olaniyan Mathew

Omar Atrooz

Raghda Hafez

Rania Amer

Rania Kishk

Rashad Abdul-Ghani

Reem Refat

Rehab El-Sokkary

Rehab Tash

Rooh Ullah

Sadek Refaat

Safaa Elnaggar

Sahbaa Fehr

Said Abbadi
Egypt

Egypt

Egypt

Sudan

Bangladesh

Bangladesh

Pakistan

Egypt

Egypt

India

Egypt

Egypt

Egypt

Egypt

Nigeria

Egypt

Egypt

Nigeria

Jordan

Egypt

Egypt

Egypt

Yemen

Egypt

Egypt

Egypt

Pakistan

Egypt

Egypt

Egypt

Egypt 


$\begin{array}{lr}\text { Salwa Seif El Dien } & \text { Egypt } \\ \text { Samar El-Beshbishi } & \text { Egypt } \\ \text { Samia Etewa } & \text { Egypt } \\ \text { Sara Abdelrahman } & \text { Egypt } \\ \text { Shoukry Mahmoud } & \text { Egypt } \\ \text { Shahenda Badran } & \text { Egypt } \\ \text { Shamshul Ansari } & \text { Nepal } \\ \text { Soma Ajlan } & \text { Egypt } \\ \text { Tadesse Eguale } & \text { Ethiopia } \\ \text { Takwa Farid } & \text { Egypt } \\ \text { Umar Abdullahi } & \text { Nigeria } \\ \text { Youssef Aboelwan } & \text { Egypt } \\ \text { Wael Abdalazem } & \text { Egypt } \\ \text { Wafaa Mosallamy } & \text { Egypt } \\ \text { Wafaa Zahran } & \text { Egypt } \\ \text { Walaa Khater } & \text { Egypt } \\ \text { Walid Faisal } & \text { Egypt }\end{array}$

